\title{
Bone and Soft Tissue Injuries in Post-mortem Lumbar Spines
}

\author{
J. R. Taylor, MB, ChB, PhD, ${ }^{1}$ L. T. Twomey, BAppl.Sci, BSc, Phd, ${ }^{2}$ \\ M. Corker BSc ${ }^{1}$ \\ ${ }^{1}$ Department of Anatomy and Human Biology, University of Western Australia, \\ Nedlands, Western Australia 6009, ${ }^{2}$ School of Physiotherapy, Curtin University \\ of Technology, Shenton Park, Western Australia.
}

\section{Summary}

A histological study of transverse sections of lumbar facet joints from 36 lumbar spines of subjects dying from serious trauma revealed injuries not visible on standard radiography, and in a high percentage of joints. They include fractures of the superior articular process, central infractions of the subchondral bone plate, and tears of the articular capsule, including the ligamentum flavum. The facet joint injuries in young subjects were almost entirely absent from the facet joints of a comparable group of young subjects with no recent history of major trauma. The injuries resemble some of the 'age changes' seen in older subjects dying non-violent deaths. It is suggested that such injuries may lead to early onset facetal arthritis in survivors of major trauma.

Key words: Spinal trauma; Lumbar facet joints; Lumbar spine injuryradiological studies; Lumbar spine injury - pathological changes.

Standard radiological investigations of patients complaining of back pain following injury, frequently reveal no bony injury. This may be taken as evidence that there is no significant injury, on the basis that any soft tissue injuries will heal within 3 to 6 weeks. Consequently, when patients continue to complain of pain after 3 to 6 months, they are suspected of manifesting a behavioural rather than an organic problem. This study describes injuries to the bones and soft tissues of lumbar facet joints, in subjects dying of major trauma, none of which were visible on standard radiological examination. These were found during a routine study of ageing in horizontally sectioned facet joints from 116 postmortem lumbar spines of subjects ranging in age from 2 years to over 80 years. It is suggested that some of these injuries could form the basis of early degenerative spondylosis.

\section{Materials and methods}

In a study of facet joints from 116 lumbar spines collected at post-mortem (Table I), spinal blocks were excised from the twelfth thoracic level to the mid-sacrum, 
Table I Numbers of joints sectioned by segmental level and age

\begin{tabular}{|c|c|c|c|c|c|c|}
\hline \multirow{2}{*}{$\begin{array}{l}\text { Age group } \\
\text { (years) }\end{array}$} & \multicolumn{2}{|c|}{ L1-2 } & \multicolumn{2}{|c|}{ L3-4 } & \multicolumn{2}{|c|}{$\mathrm{L} 4-5^{+}$} \\
\hline & Trauma & Non- $T^{\star}$ & Trauma & Non- $T^{\star}$ & Trauma & Non- $T^{\star}$ \\
\hline $2-12$ & 6 & 13 & 6 & 10 & 6 & 14 \\
\hline $13-19$ & 14 & 6 & 18 & 6 & 22 & 6 \\
\hline $20-35$ & 10 & 16 & 11 & 12 & 14 & 16 \\
\hline $36-59$ & & 25 & & 20 & & 25 \\
\hline $60+$ & $3 \#$ & 23 & $2 \#$ & 23 & $3 \#$ & 21 \\
\hline Totals & 33 & 83 & 37 & 71 & 45 & 82 \\
\hline
\end{tabular}

^ Non- $\mathrm{T}=$ no recent history of trauma

\# the last two age groups are treated together in the Trauma column

${ }^{+}$L5-S1 joints from 3 spines are included with L4-5 joints

together with the post-spinal muscles. Standard antero-posterior and lateral radiographs were taken of each lumbar spine and any spines showing bony injury were excluded from the study. The spines were fixed for 2 weeks in $10 \%$ formalin. Individual facet joints with their superior and inferior recesses were excised together with the posterior third of the corresponding disc and vertebral bodies. Each block included the pedicle of the lower vertebra and the lower margin of the upper pedicle so as to preserve the joint's relationships with the intervertebral foramen, the spinal canal and the anterior elements. The L1-2 and L4-5 joints from one side were embedded in 5\% gelatin, deep frozen overnight at $-24{ }^{\circ} \mathrm{C}$ and transversely sectioned at $2 \mathrm{~mm}$ thickness on a band saw provided with a precision guide, to be mounted in perspex trays in Wentworth No. 2 solution for low power microscopic examination. The L3-4 joint from one side was post fixed for 1 week in Bouin's fluid, decalcified in formic acid/ citrate (Taylor, 1973) and embedded in low viscosity nitrocellulose, to be sectioned at 100 microns, using a Reichert-Jung sledge microtome. One in every 7 consecutive sections was stained with haematoxylin and light green and mounted on a glass slide in Eukitt. All joints throughout the wide age range collected, were studied to establish the 'normal pattern' of age changes in the articular cartilage and capsular tissues (Taylor and Twomey, 1986). Measurements of capsular and articular cartilage thickness were made using Mitutoyo calipers at $\times 5$ magnification and all examples of capsular and ligamentum flavum damage were noted on $\times 10$ and $\times 40$ microscopic examination using a Leitz Dialux microscope.

In spite of the care taken to exclude any spines with bony injury from the age change study, a number of joints showed fractures of the superior articular process (SAP) or central infractions of one of the articular surfaces.

Since these joints came from young subjects who had died as a result of motor vehicle accidents, all zygapophyseal joints from subjects dying as a consequence of major trauma, were reexamined. The results of these observations are the principal subject of this account, i.e. the prevalence of bony injury and joint capsular damage in the joints from 36 spines of subjects dying of major trauma are compared with the prevalence of these injuries in the remaining 83 subjects. 


\section{Observations and discussion}

\section{Description of relevant articular anatomy}

Each lumbar facet joint is formed by the articulation of the convex articular surface of the inferior articular process (IAP) of the vertebra above with the concave articular surface of the superior articular process (SAP) of the vertebra below. The SAP faces posteromedially and the IAP faces anterolaterally. The posterior aspect of the joint is enclosed by a $1 \mathrm{~mm}$ thick fibrous capsule and the anteromedial aspect is covered by the elastic ligamentum flavum, which is about $3 \mathrm{~mm}$ thick at the joint line. The ligamentum flavum has an extensive medial attachment to the anterior surface of each IAP and it passes laterally to wrap around the anterior margin of the SAP, leaving only a small joint recess extending up to 2 or $3 \mathrm{~mm}$ on each side of the joint line, down the anterior margin of each articular process. The deep part of the posterior capsule is attached close to the articular margins, or directly into the posterior margin of the articular cartilage on the SAP; the superficial part extends from the mammillary process of the SAP, across the posterior surface of the IAP, to attach about $7 \mathrm{~mm}$ medial to the joint line. The two parts are partly separated by an adipose layer. The anterior elastic ligament and the posterior fibrous capsule merge with one another at the superior and inferior joint recesses. The articular cartilage lining each facet is about $1 \mathrm{~mm}$ thick.

\section{Soft tissue damage in subjects dying of major trauma}

Posterior fibrous capsule. Tears or strains of the posterior fibrous capsule, with buckling or convolution of its fibres, are increasingly common with increase in age, in adult subjects dying of non-traumatic causes. Such damage is entirely absent in subjects under 13 years with no history of recent trauma and only 1 example of capsular damage is found in an 'adolescent' in the 13 to 19 year age group. By contrast, it is unusual to find an undamaged fibrous capsule in subjects over 60 years. The capsular damage may involve tears of the fibrous capsule, separation of capsular fibres from their normal points of attachment, or damage to the articular cartilage at the point of capsular attachment, with detachment of the posterior marginal articular cartilage (Fig. 1). Alternatively, there may be gross stretching of the capsule with buckling of its fibres or wide separation of the posterior parts of the articular surfaces. These changes are most obvious in the lower part of the joint and at the inferior recess. The damage to the posterior articular margin, is related to the observation that the deep fibres of the fibrous capsule are normally attached directly into the articular cartilage margin of the SAP.

Ligamentum flavum. As an age change, unrelated to recent major trauma, a number of older joints show the ligamentum flavum partly separated from its usual extensive attachment to the anterior IAP surface. This separation may be associated with posterior capsular damage and accompanied by partial posterior subluxation of the IAP from the SAP. Such changes are not seen in any of the children or adolescents dying 'non-violent' deaths, but in a number of young subjects dying of major trauma, frank tears of the ligamentum flavum, or wide 


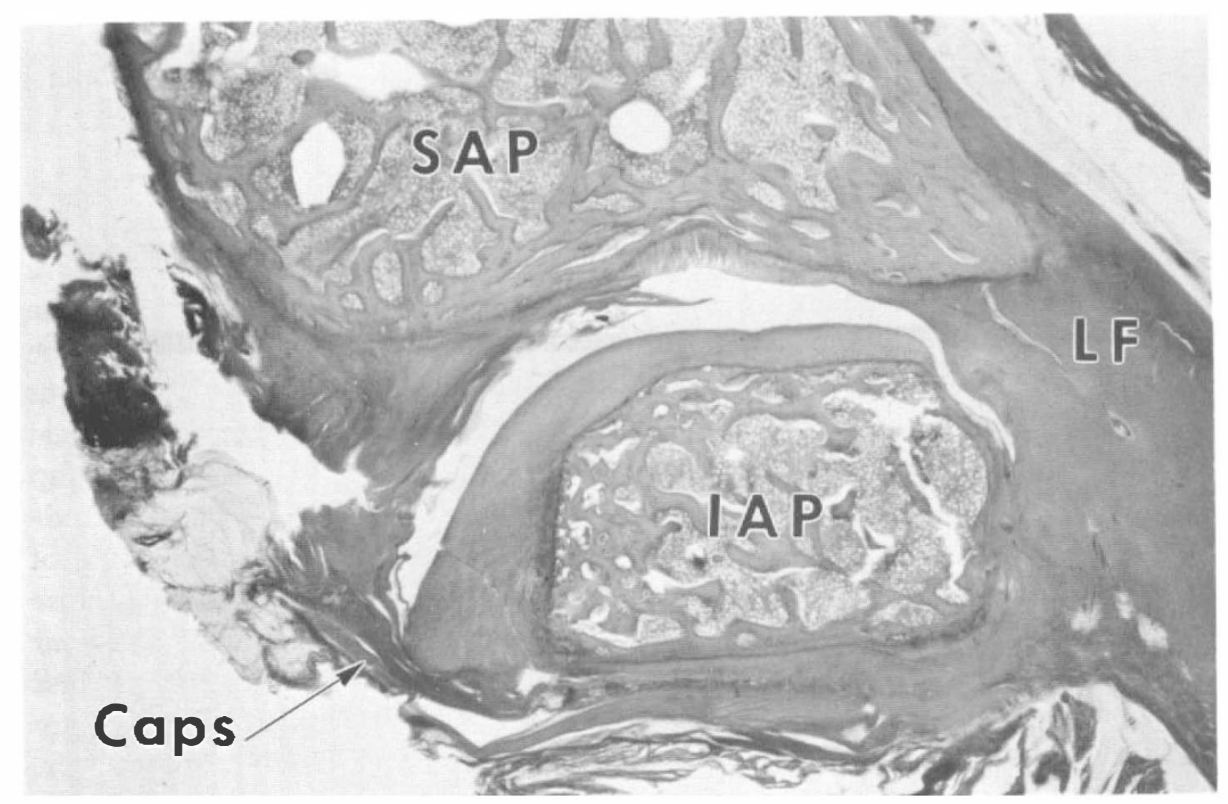

Figure 1 A 100 micron transverse section, with haematoxylin and light green, sectioned through the lower third of an LVN embedded, left L3-4 facet joint from a 7-year-old female who died as a result of a motor vehicle accident. The posterior fibrous capsule is torn at its attachment to the unossified mammillary process of the SAP. There is also damage to the central articular surface of the SAP and to the posterior articular margin of the IAP.

$\mathrm{SAP}=$ superior articular process: IAP $=$ inferior articular process.

$\mathrm{LF}=$ ligamentum flavum; Caps $=$ fibrous capsule.

Table II Capsular damage in deaths from trauma and deaths from other causes: percentage of joints affected

\begin{tabular}{|c|c|c|c|c|c|c|}
\hline \multirow{2}{*}{$\begin{array}{l}\text { Age group } \\
\text { (years) }\end{array}$} & \multicolumn{2}{|c|}{ L1-2 } & \multicolumn{2}{|c|}{ L3-4 } & \multicolumn{2}{|c|}{ L4-5 ${ }^{+}$} \\
\hline & Trauma & Non- $T^{\star}$ & Trauma & Non- $T^{\star}$ & Trauma & Non- $T^{\star}$ \\
\hline $2-12$ & $67^{\bullet}$ & 0 & $67^{\circ}{ }_{0}$ & 0 & $33^{\circ}{ }_{0}$ & 0 \\
\hline $13-19$ & $36^{\circ} \%$ & 0 & $39^{\circ}{ }_{0}^{\prime \prime}$ & $17^{\circ}{ }_{0}$ & $36^{\circ}{ }_{0}^{\circ}$ & 0 \\
\hline $20-35$ & $50 \%$ & $43^{\circ}{ }_{0}$ & $64^{\circ}{ }_{0}$ & $44^{\circ}{ }_{0}$ & $50^{\circ}{ }_{0}$ & $55^{\circ}{ }_{0}$ \\
\hline $36-59$ & & $56^{\circ}{ }_{0}$ & & $79^{\circ}{ }_{0}^{\circ}$ & & $73^{\circ}{ }_{0}$ \\
\hline $60+$ & $100^{\circ}$ & $86^{\circ}{ }_{0}$ & $100^{\circ}{ }_{0} \#$ & $86^{\circ}{ }_{0}$ & $67^{\circ}{ }_{0}^{\circ} \#$ & $83^{\circ}{ }_{0}$ \\
\hline
\end{tabular}

^ Deaths from causes other than major trauma

\# the last two age groups are treated together in the Trauma column

${ }^{+}$L5-S1 joints from 3 spines are included with L4-5 joints

separation of the ligamentum flavum from its IAP attachment is found (Fig. 2).

In summary, damage to the fibrous capsule or ligamentum flavum is found in only 1 of 20 young subjects dying from non traumatic causes, but in 17 of the 22 young subjects dying from major trauma, there is damage to the capsule or ligamentum flavum. The data on capsular damage is expressed as the percentage of joints examined showing such damage. The tears were sometimes associated with bruising in the adjacent tissue, or with blood in the extradural space; the damage could always be followed through consecutive sections, sometimes extending down the greater part of the anterior or posterior joint margin. 


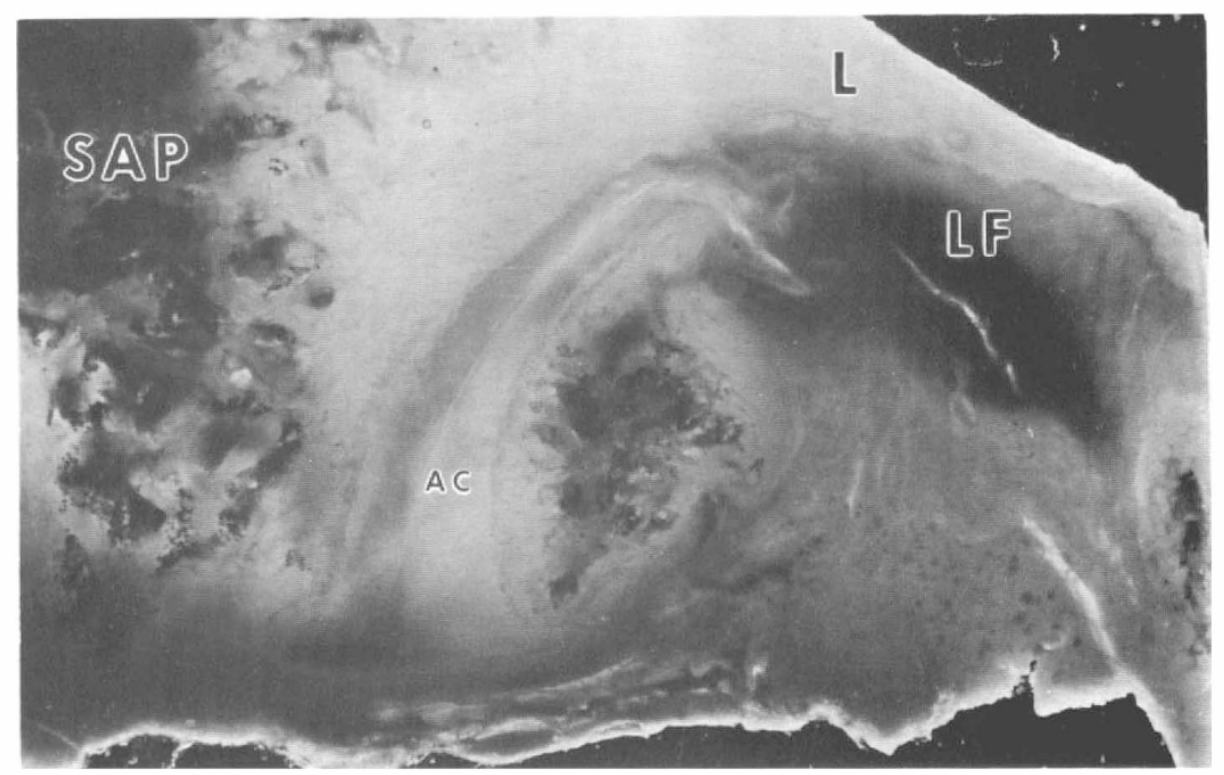

Figure 2 A 2 mm thick, unstained, undecalcified transverse section of the left L1-2 facet joint near the inferior recess, showing an extensive tear in the ligamentum flavum with dark blood staining of the ligament around the tear. SAP $=$ superior articular process. $\mathrm{LF}=$ ligamentum flavum; $\mathrm{L}=$ lamina; $\mathrm{AC}=$ articular cartilage of IAP.

Table III Bony injuries in deaths from trauma and deaths from other causes: percentage of joints affected

\begin{tabular}{|c|c|c|c|c|c|c|}
\hline \multirow{2}{*}{$\begin{array}{l}\text { Age group } \\
\text { (years) }\end{array}$} & \multicolumn{2}{|c|}{ L1-2 } & \multicolumn{2}{|c|}{ L3-4 } & \multicolumn{2}{|c|}{ L4-5 ${ }^{+}$} \\
\hline & Trauma & Non- $T^{\star}$ & Trauma & Non- $T^{\star}$ & Trauma & Non- $T^{\star}$ \\
\hline $2-12$ & $17_{0}^{\circ}$ & 0 & $33^{\circ}{ }_{0}$ & 0 & $17 \%$ & 0 \\
\hline $13-19$ & $21^{\circ}$ & 0 & $11 \%$ & 0 & $14 \%$ & 0 \\
\hline $20-35$ & $10^{\circ} \%$ & $6^{\circ}{ }_{0}$ & $18^{\circ}{ }_{0}^{\circ}$ & $16 \%$ & 0 & 0 \\
\hline $36-59$ & & 0 & & $15 \%$ & & 0 \\
\hline $60+$ & $33^{\circ}{ }_{0} \#$ & 0 & $100^{\circ}{ }_{0} \#$ & $9 \%$ & 0\# & 0 \\
\hline
\end{tabular}

$\star$ Deaths from causes other than major trauma

\# the last two age groups are treated together in the Trauma column

${ }^{+}$L5-S1 joints from 3 spines are included with L4-5 joints

\section{Bony injuries}

Fifteen of the 36 individuals show fractures or infractions of articular processes. The 9 fractures in 8 individuals, involve the SAP: the 9 small infractions in 8 individuals, involve the central parts of the subchondral bone plates (SCP) of either the SAP or the IAP. Three individuals show bony injuries to 2 of the 3 joints examined (Table III).

In the skeletally immature individuals ( $4-15$ years), the bony injuries ( 3 central 


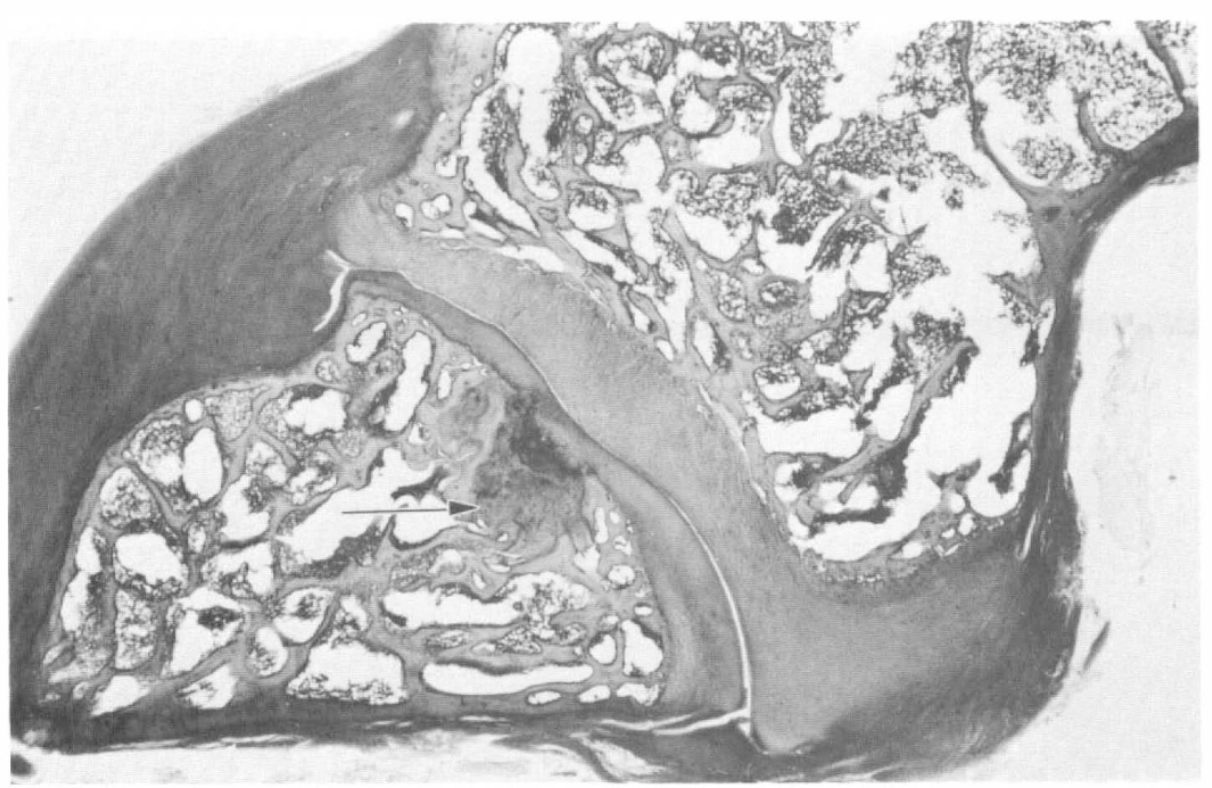

Figure 3A A transverse 100 micron section, through the lower half of a right L3-4 joint of a 7 year male who died 10 days after a motor vehicle accident, showing an infraction of the central IAP (arrowed); stained haematoxylin and light green.

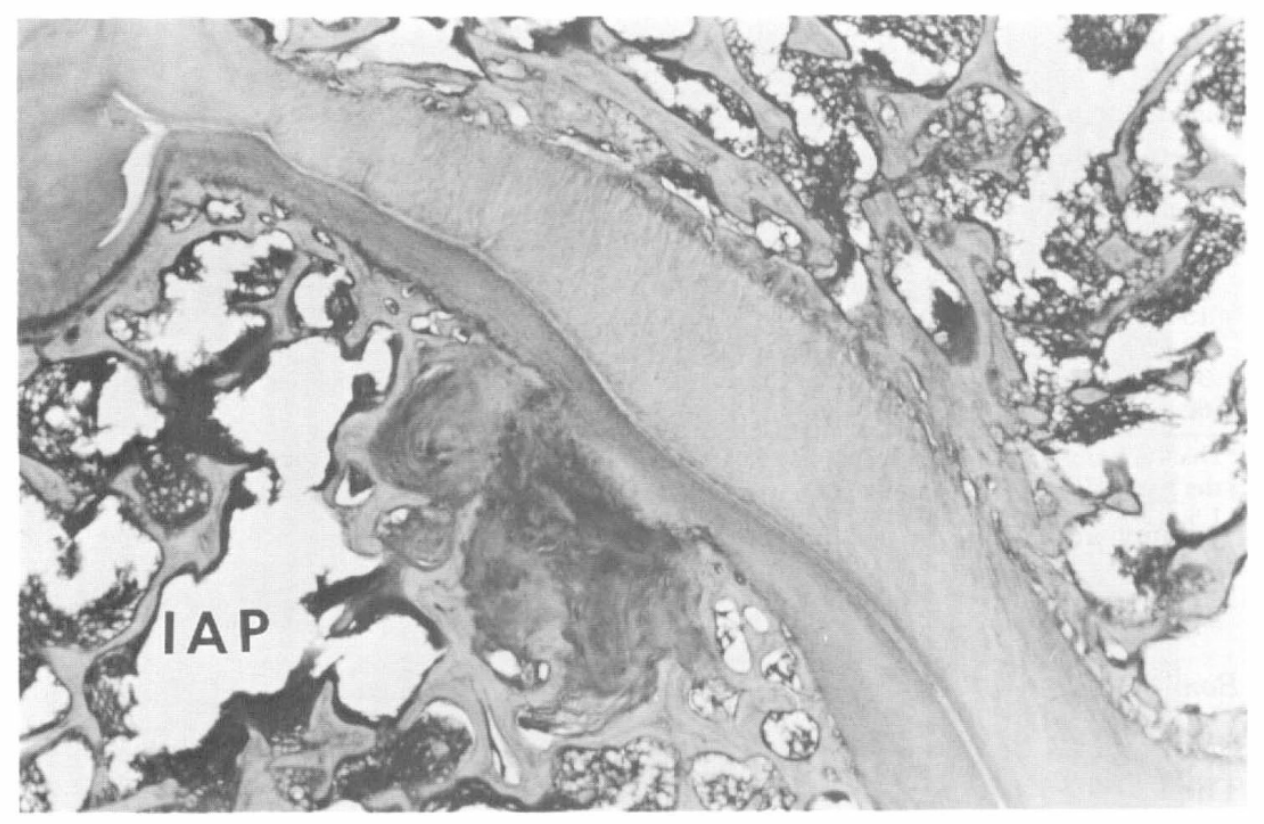

Figure 3B The central and anterior areas of the next section at higher power, showing an organising blood clot, deep to the infracted area in the IAP.

IAP $=$ inferior articular process. 


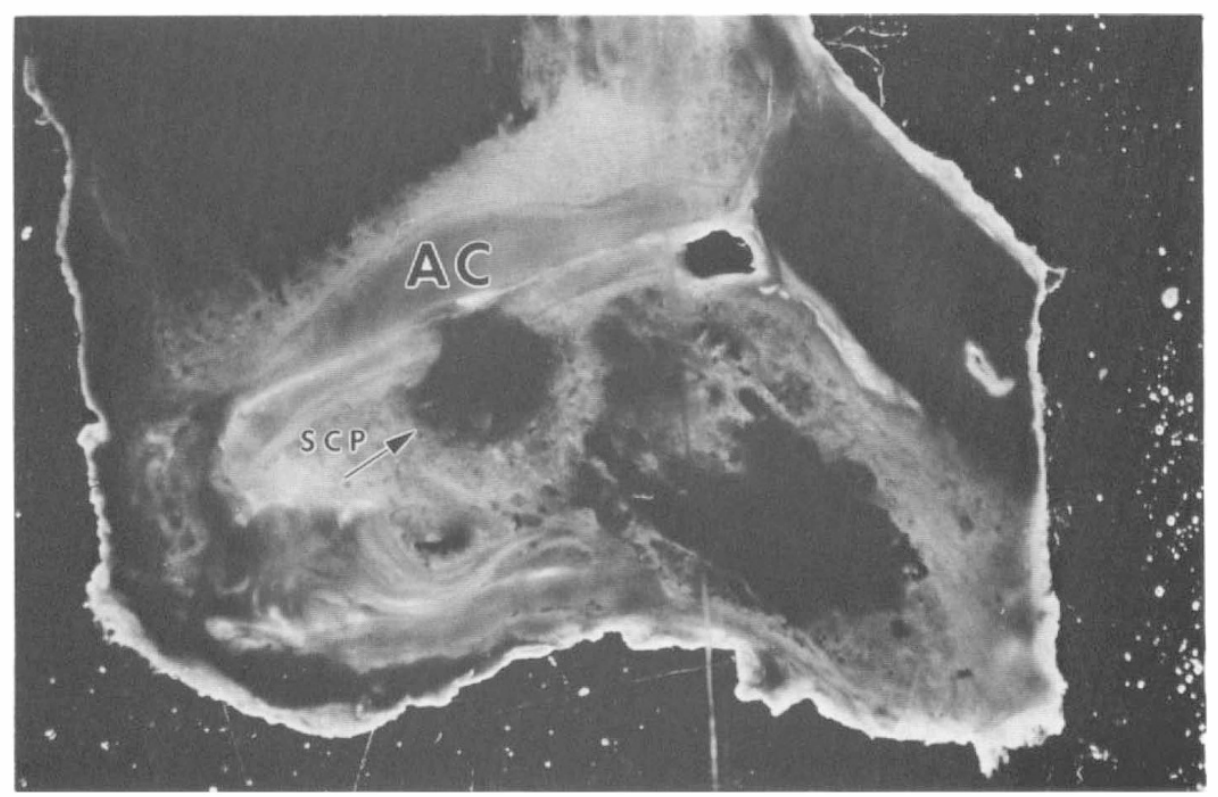

Figure 4 A 2 mm, unstained, undecalcified, mid-joint, transverse section, (dark ground illumination) from a 53-year-old female who died of major trauma. Note the bleeding into the subchondral bone of the central IAP and some blood in the joint.

$\mathrm{SCP}=$ subchondral bone plate of superior articular process; $\mathrm{AC}=$ articular cartilage.

fractures through the SAP and 4 central infractions of the SAPs or IAPs) are all 'centrally situated' in the joint and appear to be flexion-compression injuries (Fig. 3). In a flexed position, the articular surfaces are not congruous, as they would be in the neutral position. The articular surface of the lower part of the IAP has a smaller radius of curvature than the articular surface of the SAP at mid-joint level. When in flexion, the more sharply curved surface of the IAP is compressed against the flatter SAP, central damage may be expected. This applies particularly to the younger individuals where the central SCP has not grown to its full thickness and strength (Taylor and Twomey, 1985).

The 6 fractures in the 16,17,19, 22 and 48-year-old subjects are laterally situated in the SAP, through the 'base' of the mammillary process (Fig 5, Fig. 6). They appear to be avulsion fractures of that part of the SAP projecting posterolateral to the congruous articular surfaces, to which the the capsule and multifidus are attached. The mechanism of production would be similar to the mechanism producing damage to the posterior joint margin, with capsular tearing or partial separation of the posterior marginal articular cartilage (Taylor and Twomey, 1986) viz., combined flexion and rotation, perhaps with reflexly tensed extensor muscles. These lateral fractures are seen in subjects where the anterior and central parts of the SCP have grown to their full thickness and strength (Taylor and Twomey, 1985). Bony injuries cannot occur in the mammillary processes of younger subjects where this part is not yet ossified and there is a cartilage growth plate. 


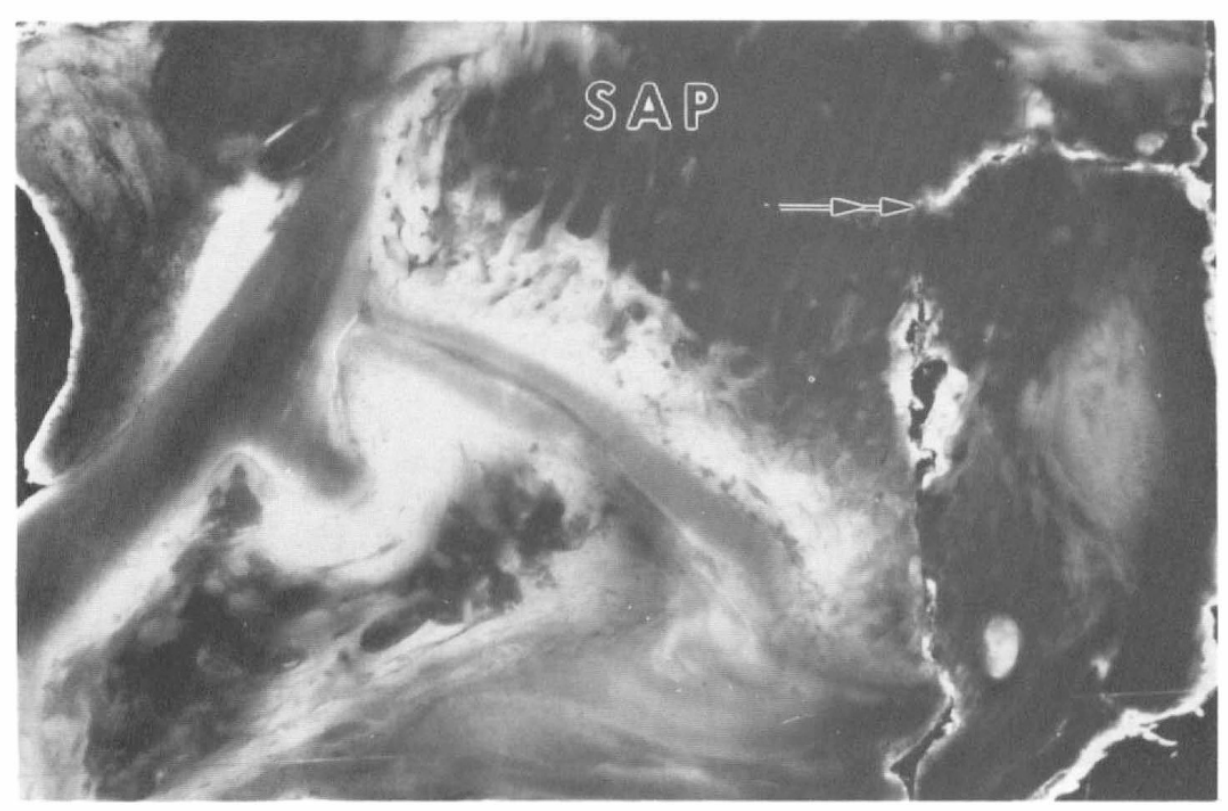

Figure 5 An unstained transverse $2 \mathrm{~mm}$, section of the right L4-5 joint, viewed by dark ground illumination, from a 17 -year-old male subject who died following a motor vehicle accident. Note the vertical, saggittal plane fracture through the lateral part of the SAP.

SAP $=$ superior articular process.

\section{Differentiation of trauma from age change and artefact}

There are at least three possible reasons for the anomalies observed in our sectioned material: artefact, age change, and acute trauma. In the stained 100 micron sections, age changes can be recognised by the histological changes, characteristic of the tissues' reaction and repair over a period of time. The frequent association of bony and capsular damage with death from major trauma, and its virtual absence in the two youngest groups who died of 'nonviolent' causes is strongly in favour of the changes in the young subjects being due to the ante-mortem trauma. The presence of blood in the joint in instances of infraction, the observation of an organising blood clot in an infraction where the child survived the accident for 10 days (Fig. 3), the presence of bruising in torn ligamentum flavum (Fig. 2) or in damaged tissues at the posterior margin of a joint, or in the extradural space, despite the frequent fluid changes associated with washing and processing the blocks, is further evidence for the ante-mortem nature of this damage. The presence of damage in the same location in numerous consecutive sections confirms its presence in the block before sectioning.

Soft tissue injuries are the most common injuries observed. Capsular or ligamentum flavum damage, as described above, is seen in one or more joints, in 33 of the 36 lumbar spines from subjects dying of major trauma. Some of this soft tissue damage may be due to age change, and minor ante-mortem damage 


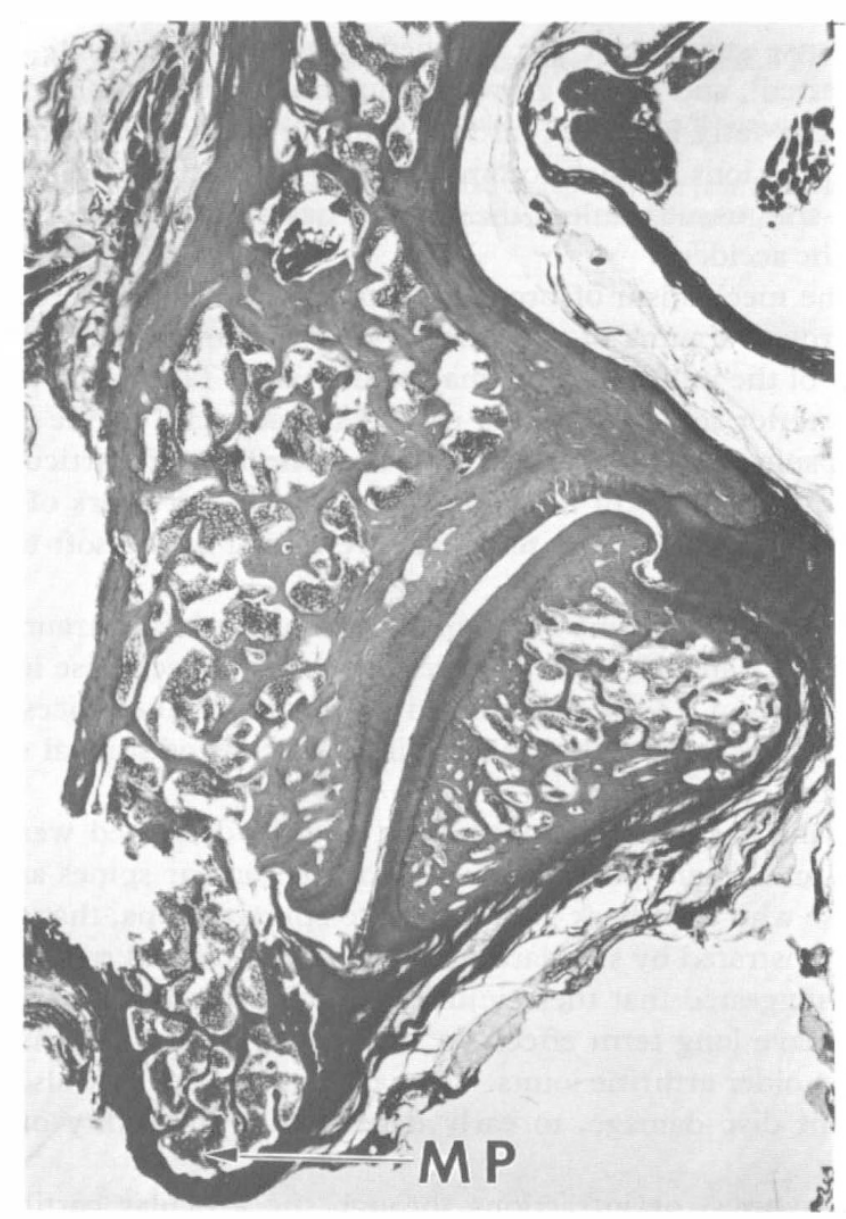

Figure 6 A 100 micron transverse section of an LVN embedded left L3-4 joint of a 19-year-old female, stained by haematoxylin and light green. Note the fracture through the base of the mammillary process of the superior articular process. $\mathrm{MP}=$ mammillary process.

may be artefactually exaggerated by processing for histology, but its virtual absence in the joints from the 20 'non-traumatic' deaths in the two youngest groups argues strongly that it cannot be accounted for by artefact alone.

Damage to the articular cartilage of the anterior part or the central concavity of the SAP, is also frequently seen, but since these lesions are characteristic of the response to the normal stresses of everyday living as seen with ageing they are not described in this study (Taylor and Twomey, 1986).

The infractions and posterior marginal damage accompanying ageing (Taylor and Twomey 1986) differ from those of very recent trauma. The longer term 'age changes' show tissue reactions such as chondrocyte swelling and intense staining of the pericellular matrix in the mid-zone of the articular cartilage. 
Capsular strains of long standing, can be distinguished by the thickening, irregularity and intense staining of the calcified cartilage zone where the capsular fibres are 'inserted', and by the growth of an abnormally large, loosely formed, space-filling fibro-fatty inclusion into the joint, in the region of the strain. The acute traumatic lesions are not accompanied by these changes in the cells and matrix of the soft tissues unless the subject has survived for some weeks or months after the accident.

However, the mechanism of production of soft tissue injury, whether acute trauma or 'chronic' trauma of age change, may be similar in nature, although the magnitude of the injuring forces may be different. The nature of the pathology at the posterior joint margin, is related in each case to the continuity of the fibrous capsule with the posterior articular margin of the articular cartilage. As a corollary, the results of acute major trauma in survivors of this trauma may be regarded as comparable to premature ageing in the soft tissues of the joints.

The prevalence of different types of injury in the 'acute trauma' group is shown in Tables II and III. The changes are easier to recognise in the stained 100 micron sections of L3-4 joints, than in the thick unstained slices of L1-2 and L4-5, but their presence in the undecalcified slices is additional confirmation that they are not artefacts.

It is important to note that none of the injuries described were visible on normal lateral and antero-posterior X-rays of the lumbar spines and similarly, to remind those who treat back pain related to spinal trauma, that such injuries cannot be demonstrated by standard methods of clinical and radiological investigation. It is suggested that these injuries, which are predominantly in young subjects, may have long term effects in survivors of trauma similar to the age changes seen in older arthritic joints. The capsular injuries may also contribute, with concurrent disc damage, to early degenerative instability of the mobile segment.

In facetal fractures, or infractions through the articular cartilage, healing may be associated with deformity of the articular surfaces, predisposing to early arthritis, with chronic pain and stiffness. It is likely that abnormal lumbar spinal posture would be more common in such people. If uncorrected by good management, dysfunction and poor posture might persist long after the initial pain due to posterior capsular damage had ceased; low back pain or discomfort are commonly associated with persistent abnormal posture.

Such individuals require to maintain muscle fitness and normal ranges of movement and to pay particular attention to good posture, whether standing, sitting or lying in bed. They also require good advice about appropriate exercise habits to improve healing and remodelling of damaged tissues. The tendency to be dismissive of patients with such 'minor' injuries is to be deprecated as much as the tendency to focus undue attention on these injuries.

\section{Acknowledgement}

The authors wish to acknowledge the financial support received for this project, from the Medical Research Fund of Western Australia. 


\section{References}

TAYLOR JR 1973 Growth and development of the human intervertebral disc. PhD Thesis, University of Edinburgh, p 41.

TAYLOR JR, TWOMEY LT 1985 Age changes in the subchondral bone of human lumbar zygapophyseal joints. Fournal of Anatomy 143:233.

TAYLOR JR, TWOMEY LT 1986 Age changes in lumbar zygapophyseal joints. Spine 11:739-745.

TwOMEy L, TAYLOR JR 1985 Age changes in lumbar intervertebral discs. Acta Orthopaedica Scandinavica 56:496-499. 